\title{
Silencing of FANCD2 enhances the radiosensitivity of metastatic cervical lymph node-derived head and neck squamous cell carcinoma HSC-4 cells
}

\author{
HUA-JUN FENG, YI-LIN BAO, ZHUO-PING LIANG, FEI-PENG ZHAO, \\ SHENG-EN XU, WEI XU, CHONG ZHAO and GANG QIN \\ Department of Otolaryngology Head and Neck Surgery, The Affiliated Hospital of \\ Southwest Medical University, Luzhou, Sichuan 646000, P.R. China
}

Received November 16, 2016; Accepted February 7, 2017

DOI: 10.3892/ijo.2017.3902

\begin{abstract}
Fanconi anemia complementation group D2 (FANCD2) is involved in the key steps of the Fanconi anemia (FA) pathway, which plays a role in the repair of DNA crosslink damage. However, the role of FANCD2 during radiotherapy for head and neck squamous cell carcinoma (HNSCC) is unclear. In this study, the HNSCC cell line HSC-4 was used. Western blotting was used to evaluate the expression of the FANCD2 in HSC-4 cells. We investigated the impact of FANCD2 on the radiosensitivity of HSC-4 cells in vitro and in vivo. TUNEL, western blotting and immunohistochemistry were used to analyze the apoptosis and proteins involved in apoptosisrelated pathways after radiotherapy to investigate the relevant mechanism. The present study showed that shRNA interference could effectively and stably silence FANCD2 expression in HSC-4 cells. In vitro, the silencing of FANCD2 inhibited cell proliferation, decreased the survival rate, increased apoptosis and induced S phase arrest in HSC-4 cells after radiotherapy. In vivo, the silencing of FANCD2 could prolong the tumor-forming time and slow tumor growth. In addition, the tumor volume was significantly reduced, the weight was deceased, and the tumor inhibition rate was increased after radiotherapy. TUNEL showed that the silencing of FANCD2 significantly increased apoptosis in HSC-4 cells induced by radiotherapy. Both in vitro and in vivo esperiments revealed that the expression of the Bax and p-p38 proteins in HSC-4 cells, in which FANCD2 had been silenced, was increased after radiotherapy, whereas the expression of the p38 and $\mathrm{Bcl} 2$ proteins was decreased. Our results suggested that the silencing of FANCD2 enhanced the radiosensitivity of HSC-4
\end{abstract}

Correspondence to: Dr Gang Qin, Department of Otolaryngology Head and Neck Surgery, The Affiliated Hospital of Southwest Medical University, No. 25 Taiping Street, Jiangyang, Luzhou, Sichuan 646000, P.R. China

E-mail: qing_lzmc@163.com

Key words: Fanconi anemia complementation group D2 protein, Fanconi anemia, head and neck cancer, HSC-4, radiosensitivity cells, and its mechanism involves the activation of the p38 MAPK signaling pathway and the regulation of the expression of $\mathrm{Bax}$ and $\mathrm{Bcl} 2$ proteins. This study provides a novel candidate target for HNSCC therapy.

\section{Introduction}

On a global scale, head and neck cancer is the sixth most common type of malignant cancer type in humans, and the major histological type is squamous cell carcinoma (1). Over 600,000 new cases of head and neck squamous cell carcinoma (HNSCC) occur each year, and most of the individuals with HNSCC are already in the advanced stage by the time of diagnosis (2). Currently, comprehensive treatment consisting of surgery plus postoperative chemoradiotherapy is still the most commonly used method to treat advanced-stage HNSCC. However, the prognosis of patients in advanced stages is not optimistic, and over $50 \%$ of patients experience relapse and distant metastasis after treatment (3). Furthermore, tumor relapse and metastasis caused by radiotherapy resistance are still the major causes of failure of HNSCC treatment (4). Therefore, investigating molecular targets that can improve the radiosensitivity of HNSCC will have clinical significance for the effective improvement of treatment efficacy and the prognosis of patients with HNSCC.

Fanconi anemia (FA) is an autosomal or X-linked recessive genetic disease and is characterized by congenital malformations, progressive bone marrow hematopoietic failure, increased chromosomal fragility and high susceptibility to tumors (5). In patients with FA, the incidence of HNSCC is significantly higher than in the average population and most patients tend to be younger (6). Patients with FA show high sensitivity to ionizing radiation and to mitomycin $\mathrm{C}$, cisplatin and other DNA cross-linking agents. To date, 18 FA-associated genes have been found $(7,8)$. Proteins encoded by these genes compose an FA signaling pathway that is involved in the repair of DNA crosslink damage. Upstream of the FA pathway is the FA core complex, which has E3 ubiquitin ligase activity and is composed of at least eight FA proteins and FA-related proteins. The FA core complex monoubiquitinates FANCD2 and FANCI (with the ID complex formed by FANCD2 and 
FANCI), thus, further mobilizing downstream FA proteins and DNA repair-related proteins; eventually, the USP1/UAF1 complex deubiquitinates the ID complex to complete the FA pathway (9-11). The function of the FA pathway involves cell cycle regulation, DNA damage and repair, apoptosis, gene transcription and the maintenance of genome stability. Inactivation of the FA pathway, which causes gene instability and impedes the repair of DNA crosslink damage, is the molecular mechanism of FA pathogenesis.

As a central link, FANCD2 connects the upstream and downstream portions of the FA pathway, and its suppression will inevitably block the transduction of FA pathway signals (12). Some scholars have found that the downregulation of FANCD2 nuclear foci formation by phenylbutyrate can enhance the sensitivity of HNSCC to cisplatin (13). Other studies have suggested that FANCD2 plays an important role in the maintenance of gene stability and DNA damage repair during radiotherapy (14). Moreover, the downregulation of FANCD2 can enhance the 'bystander effect', which occurs during radiotherapy (15). Therefore, suppressing the role of FANCD2 and then downregulating the activity of the FA pathway may enhance the sensitivity of HNSCC to radiotherapy. However, the relationship between FANCD2 and the sensitivity of HNSCC to radiotherapy is currently unclear.

In the present study, we used shRNA to knock down the expression of FANCD2 in the metastatic cervical lymph node-derived HNSCC cell line HSC-4. Through in vitro and in vivo experiments, we investigated whether the silencing of FANCD2 could enhance the sensitivity of HSC-4 cells to radiotherapy; we also determined the associated mechanism, which has provided a new molecular target for the clinical treatment of head and neck tumors.

\section{Materials and methods}

Cell lines and cell culture. The HNSCC cell line HSC-4 was used. An experimental group FANCD2-shRNA (HSC-4 cells transfected with an effective FANCD2 interference sequence where the silencing effect is stable), negative control group FANCD2-shRNA-C (HSC-4 cells transfected with an ineffective FANCD2 interference sequence) and blank control group HSC-4 (wild-type HSC-4 cells without any treatment) were used. These cells were generously provided by Dr Stephan M. Feller of the Weatherall Institute of Molecular Medicine at the University of Oxford, UK. The cell culture conditions were as follows: cells were cultured in high-glucose Dulbecco's modified Eagle's medium (DMEM; Thermo Fisher Scientific, Beijing, China) supplemented with $10 \%$ fetal bovine serum (FBS; Bio-Cell, Beijing, China), $100 \mu \mathrm{g} / \mathrm{ml}$ streptomycin and $100 \mathrm{U} / \mathrm{ml}$ penicillin. The cells were maintained in a thermostat incubator at $37^{\circ} \mathrm{C}$ in an atmosphere of $5 \% \mathrm{CO}_{2}$ and saturated humidity. Puromycin (InvivoGen, San Diego, CA, USA), at a final concentration of $2 \mu \mathrm{g} / \mathrm{ml}$, was added to the cells of the experimental group and the negative control group as a selection reagent.

Radiotherapy. The radiotherapy for both cells and animals was conducted at room temperature using the medical linear electron accelerator Elekta Precise NET (Elekta, Stockholm, Sweden) at a dose rate of $200 \mathrm{cGy} / \mathrm{min}$.
Cell proliferation analysis. Cell Counting kit-8 (CCK-8; Sigma-Aldrich, St. Louis, MO, USA) was used to evaluate the impact of FANCD2 shRNA interference on the in vitro proliferation of HSC-4 cells after radiotherapy. Cells in the logarithmic growth phase from the three groups were seeded in 96-well plates at a density of 3,000 cells/well. Radiotherapy of $0,2,5$ or 8 Gy was given to the cells, and $48 \mathrm{~h}$ after radiotherapy, the absorbance at $450 \mathrm{~nm}$ was measured. In addition, the three groups of cells received $5 \mathrm{~Gy}$ of radiotherapy, and the absorbance at $450 \mathrm{~nm}$ was measured at 24, 48 and $72 \mathrm{~h}$ after radiotherapy, which enabled the calculation of the inhibition of the cell proliferation rate. The inhibition of the cell proliferation rate $=(1-$ average optical density $(O D)$ value of the treated group/average OD value of the blank control group) $\mathrm{x} 100 \%$. Finally, the concentration-inhibition rate curve was plotted.

Colony formation assay. A colony formation assay was used to examine the impact of FANCD2 shRNA interference on the survival rate of HSC-4 cells after radiotherapy. Cells from the three groups were seeded into $6-\mathrm{cm}$ culture plates at a predetermined number; and $24 \mathrm{~h}$ after incubation, the cells received $0,2,5$ or 8 Gy of radiotherapy, after which the cells were routinely cultured for 13 days. Then, the cells were fixed in methanol and stained with $1 \%$ crystal violet-ethanol solution. The number of clones that contained $>50$ cells was counted under a microscope, and the colony formation rate (colony formation rate $=$ number of clones/number of cells seeded $\mathrm{x} 100 \%$ ) and survival rate (survival rate $=$ the number of clones in the experimental group under a certain dose/the number of cells seeded in this group $\mathrm{x}$ colony formation rate) were calculated. The analysis was performed using the average value of the survival rate found in three different radiation treatments.

Cell apoptosis analysis. Flow cytometry was used to examine the impact of FANCD2 shRNA interference on the apoptosis rate of HSC-4 cells after radiotherapy. Cells in the logarithmic growth phase from the three groups were seeded in 6-well plates at a final concentration of $1 \times 10^{5}$ cells $/ \mathrm{ml}$; when the cells became adherent and reached a confluence of $\sim 80 \%$, they received an 8-Gy dose of radiotherapy. After radiotherapy, the cells were further cultured for $48 \mathrm{~h}$ before the experimental cells were harvested. The cells were washed twice with buffer, and cells were assayed on a machine according to the manufacturer's instructions for the Annexin V-FITC apoptosis detection kit (Nanjing KeyGen Biotech, Co., Ltd., Nanjing, China). The rate of apoptosis of the cells in each group was calculated based on the data obtained.

Cell cycle analysis. Flow cytometry was used to examine the impact of FANCD2 shRNA interference on the cell cycle distribution of HSC-4 cells after the radiotherapy. Cells in the logarithmic growth phase from the three groups received either 0 or $8 \mathrm{~Gy}$ of radiotherapy and then, the cells were harvested $48 \mathrm{~h}$ after the radiotherapy. The cells were washed twice with phosphate-buffered saline (PBS) and were assayed on the machine according to the manufacturer's instructions for the cell cycle assay kit (Nanjing KeyGen Biotech). The percentage of cells in each phase of the cell cycle was then calculated. 
Animal experiments. Female BALB/c-nu nude mice that were 3 to 4 weeks old and that weighed $18-22 \mathrm{~g}$ were purchased from Beijing HFK Bioscience, Co., Ltd., Beijing, China. The mice were housed in the specific pathogen-free (SPF) animal room of the Department of Oncology of the Affiliated Hospital of Southwest Medical University. All animal experimental procedures were performed in accordance with the appropriate institution or the National Research Council Guide for the care and use of laboratory animals and approved by the Affiliated Hospital of Southwest Medical University. The nude mice were randomly divided into three groups, with five mice in each group. Cells in the logarithmic growth phase from the three groups were used to prepare cell suspensions in highglucose DMEM at a concentration of $1.0 \times 10^{7}$ cells $/ \mathrm{ml}$. Then, $0.1 \mathrm{ml}$ of the cell suspension was subcutaneously injected into the outer side of the left thigh of the corresponding mice, and the tumor formation rate and the tumor formation time of each group were monitored. After visible tumors formed, the tumors were measured every three days using a Vernier caliper, and the tumor volume was calculated according to the formula $\mathrm{V}=\pi / 6 \times \mathrm{ax} \mathrm{b}^{2}$, where $\mathrm{a}$ is the longest diameter of the tumor, and $\mathrm{b}$ is the short diameter perpendicular to $\mathrm{a}$. When the longest diameter of the tumor was $\sim 4-6 \mathrm{~mm}$, the radiotherapy regimen commenced, which involved the local administration of radiotherapy to the tumor. According to the conventional fractionated radiotherapy method, 2 Gy was given once per day for five days for a total dose of $10 \mathrm{~Gy}$. On the 10th day after the radiotherapy was terminated, the mice were sacrificed by cervical dislocation, the tumors were removed and weighed, and the tumor inhibition rate was calculated. Each tumor was equally divided into two halves: one half was stored in liquid nitrogen for western blot analysis, while the other half was fixed in neutral-buffered formalin and paraffin-embedded for hematoxylin and eosin (H\&E) staining, terminal deoxynucleotidyl transferase-mediated dUTP nick end labeling (TUNEL) staining and immunohistochemistry.

TUNEL staining assay. TUNEL staining was used to examine tumor cell apoptosis in the three groups of cells after the radiotherapy. The protocol was performed according to the instructions of the TUNEL apoptosis assay kit (Roche Diagnostics, Shanghai, China). When the nucleus was stained brown, the cell was defined as an apoptotic cell. Under a microscope at a magnification of $\mathrm{x} 400,10$ fields were randomly selected from each slide to calculate the total number of apoptotic cells. The apoptotic index (AI) was calculated, where the $\mathrm{AI}=$ the total number of apoptotic cells/the total number of nucleated cells x $100 \%$.

Western blot analysis. Western blotting was used to determine the expression level of FANCD2 protein in HSC- 4 cells and the expression of apoptosis pathway-related proteins in the tumors and in HSC-4 cells after radiotherapy. Tumor tissue was thoroughly ground in liquid nitrogen and lysis buffer was added. The tumor tissue and buffer were mixed well, the sample was centrifuged and the proteins were extracted. Cells in the logarithmic growth phase from the three groups were seeded in $10-\mathrm{cm}$ culture plates, and a radiotherapy dose of either 0 or 8 Gy was given to each cell group. After radiotherapy, the cells were cultured for $24 \mathrm{~h}$ before they were harvested. The cells were rinsed two to three times with pre-chilled PBS and the proteins were extracted. The protein samples were adjusted to the same concentration, loaded onto a $10 \%$ sodium dodecyl sulfate polyacrylamide gel electrophoresis (SDS-PAGE) gel to separate the proteins and then transferred to a nitrocellulose membrane, which was blocked with Tris-buffered saline with Tween-20 (TBST) containing 5\% fat-free milk. The following primary antibodies were used: mouse anti-human FANCD2 polyclonal antibody diluted 1:300, rabbit anti-human Bax polyclonal antibody diluted 1:200, mouse anti-human Bcl12 monoclonal antibody diluted 1:200, mouse anti-human p38 monoclonal antibody diluted 1:200, rabbit anti-human p-p38 polyclonal antibody diluted 1:200 and mouse anti-human $\beta$-actin monoclonal antibody diluted 1:1000 (all purchased from Santa Cruz Biotechnology, Santa Cruz, CA, USA). The membrane was incubated with the primary antibody overnight at $4^{\circ} \mathrm{C}$. TBST was used to wash the membrane, and secondary antibody was prepared as follows: goat antirabbit IgG diluted 1:2,000 and goat anti-mouse IgG diluted 1:2,000 (both purchased from Santa Cruz Biotechnology). The membrane was incubated with the secondary antibody at room temperature for $1 \mathrm{~h}$. TBST was used to wash the membrane, and enhanced chemiluminescence (ECL) solution was used to develop the protein bands.

Immunohistochemistry. The EnVision ${ }^{\mathrm{TM}}$ method was used for immunohistochemistry to determine the expression levels of apoptosis pathway-related proteins in the tumors after radiotherapy. Paraffin-embedded tumors were continuously sectioned at a thickness of $4 \mu \mathrm{m}$. Sections were routinely deparaffinized in xylene and rehydrated in graded ethanol solutions. Sections were then immersed into an appropriate amount of ethylenediaminetetraacetic acid (EDTA) solution (pH 9.0) in a slide box, which was placed inside a pressure cooker for antigen retrieval under high temperature and pressure. The sections were incubated with $3 \% \mathrm{H}_{2} \mathrm{O}_{2}$ in methanol for $10 \mathrm{~min}$ at room temperature to quench the endogenous peroxidase activity. The primary antibodies were prepared as follows: rabbit anti-human Bax polyclonal antibody was diluted 1:100, mouse anti-human $\mathrm{Bcl} 2$ monoclonal antibody was diluted 1:100, mouse anti-human p38 monoclonal antibody was diluted 1:100, and rabbit anti-human p-p38 polyclonal antibody was diluted 1:100 (all purchased from Santa Cruz Biotechnology). Finally, the sections were incubated with the appropriate secondary antibody, developed with diaminobenzidine (DAB), counterstained in hematoxylin, dehydrated, cleared in xylene and mounted.

Statistical analysis. SPSS 19.0 statistical analysis software and GraphPad Prism 5 software were used to analyze the experimental results. Experimental data are represented as mean \pm SD and a one-way ANOVA was used for data comparison. $\mathrm{P}<0.05$ were considered statistically significant.

\section{Results}

shRNA interference caused a reduction in FANCD2 expression in HSC-4 cells. shRNA was used to silence FANCD2 expression in HSC-4 cells, while western blot analysis was used to examine FANCD2 expression in the three groups of 
A

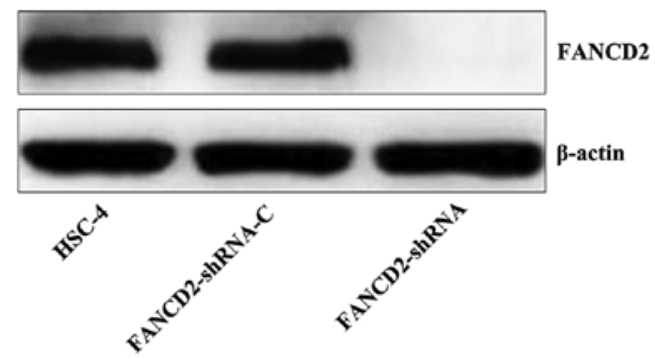

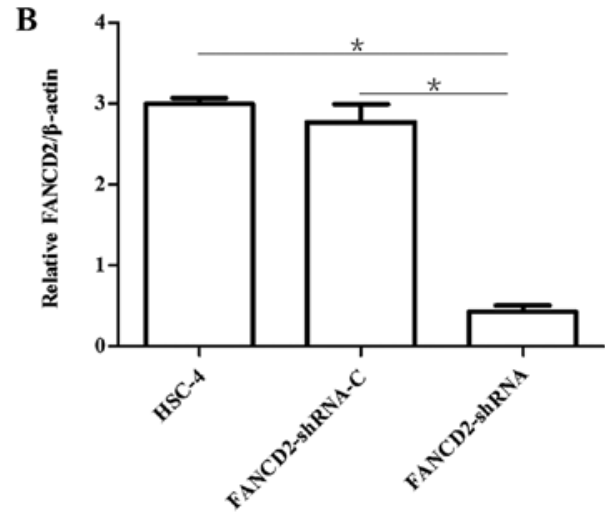

Figure 1.FANCD2 gene expression was silenced by shRNA interference in HSC-4 cells. (A) Western blot analysis shows the FANCD2 protein expression level in the three groups of HSC-4 cells. (B) Quantitative analysis of the relative expression level of FANCD2/ $\beta$-actin in the three groups of HSC-4 cells. ${ }^{*}<0.05$.
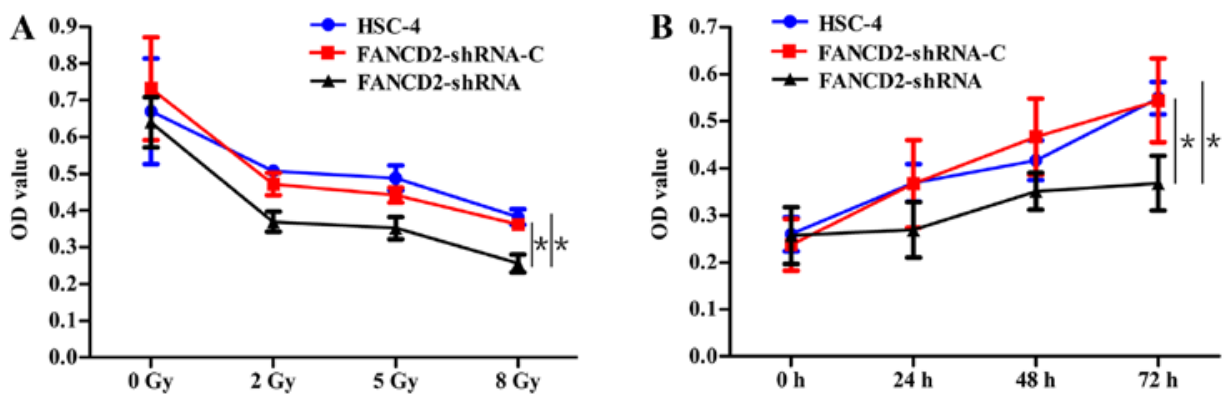

Figure 2. Cell proliferation analysis using the CCK-8 assay. Silencing of FANCD2 enhanced the proliferation inhibition effect in HSC-4 cells after radiotherapy, and the effects were dependent on dose and time. (A) OD values at $48 \mathrm{~h}$ after HSC- 4 cells were exposed to various doses of radiation. (B) OD values at different time-points after HSC-4 cells were exposed to 5 Gy of radiation. " $\mathrm{P}<0.05$.

cells. The results showed that the level of FANCD2 expression in the experimental group was significantly lower than the levels in the negative and blank control groups (P<0.05; Fig. 1), which suggests that shRNA interference can effectively and stably knock down FANCD2 gene expression in HSC-4 cells.

Silencing of FANCD2 enhanced the in vitro proliferation inhibition effect in HSC-4 cells after radiotherapy. Cell proliferation experiments showed that the proliferation levels of cells in all three groups that received radiotherapy were lower than those in the cells that did not receive radiotherapy $(\mathrm{P}<0.05)$. Moreover, the proliferation inhibition effect was enhanced as the radiation dose increased. The proliferation rate of the HSC-4 cells in the experimental group was lower than that of the control groups after radiotherapy with various dose $(\mathrm{P}<0.05)$, and the proliferation inhibition effect was the most significant when the radiation dose was 8 Gy (Fig. 2A), which suggests that the silencing of FANCD2 gene expression can enhance the effect of proliferation inhibition in HSC-4 cells after radiotherapy and that this effect is dose-dependent. When the radiation dose was $5 \mathrm{~Gy}$ for the three groups of cells, the proliferation inhibition effect of the experimental group was significantly enhanced compared with that of the control groups at the three time-points of 24, 48 and $72 \mathrm{~h}(\mathrm{P}<0.05$; Fig. 2B). This finding suggests that the silencing of FANCD2 gene expression can enhance the effects of proliferation inhibition of HSC- 4 cells after radiotherapy and that this effect is time-dependent.

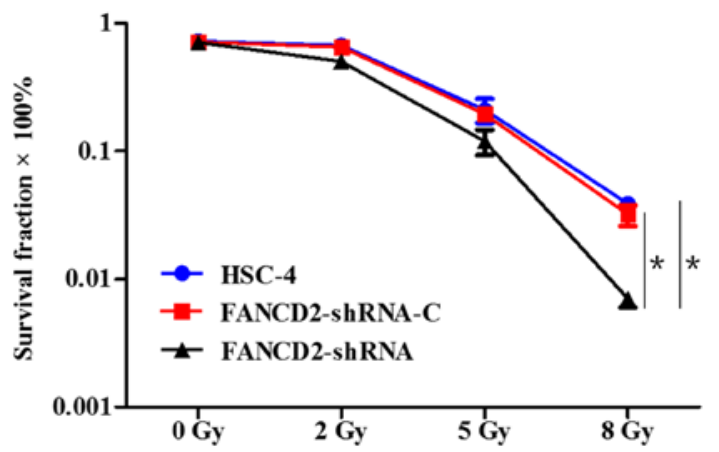

Figure 3. Silencing of FANCD2 reduced the survival rate of HSC-4 cells after radiotherapy. The cell survival rate of the three groups of HSC-4 cells was determined using a colony formation assay 13 days after exposure to 0 , 2,5 and 8 Gy of radiotherapy. ${ }^{*} \mathrm{P}<0.05$.

Silencing of FANCD2 reduced the survival rate of $\mathrm{HSC}-4$ cells after radiotherapy. The colony formation assay showed that the survival rates of the three groups of cells were not significantly different before radiotherapy was administered ( $\mathrm{P}>0.05$ ), but as the radiotherapy dose increased, the survival rate of cells in each group decreased. When the dose of radiotherapy was $8 \mathrm{~Gy}$, the survival rate of cells in the experimental group was significantly lower than that of cells in the negative control and blank control groups $(\mathrm{P}<0.05$; Fig. 3), which suggests that the silencing of FANCD2 reduced the survival rate of HSC-4 cells after radiotherapy. 

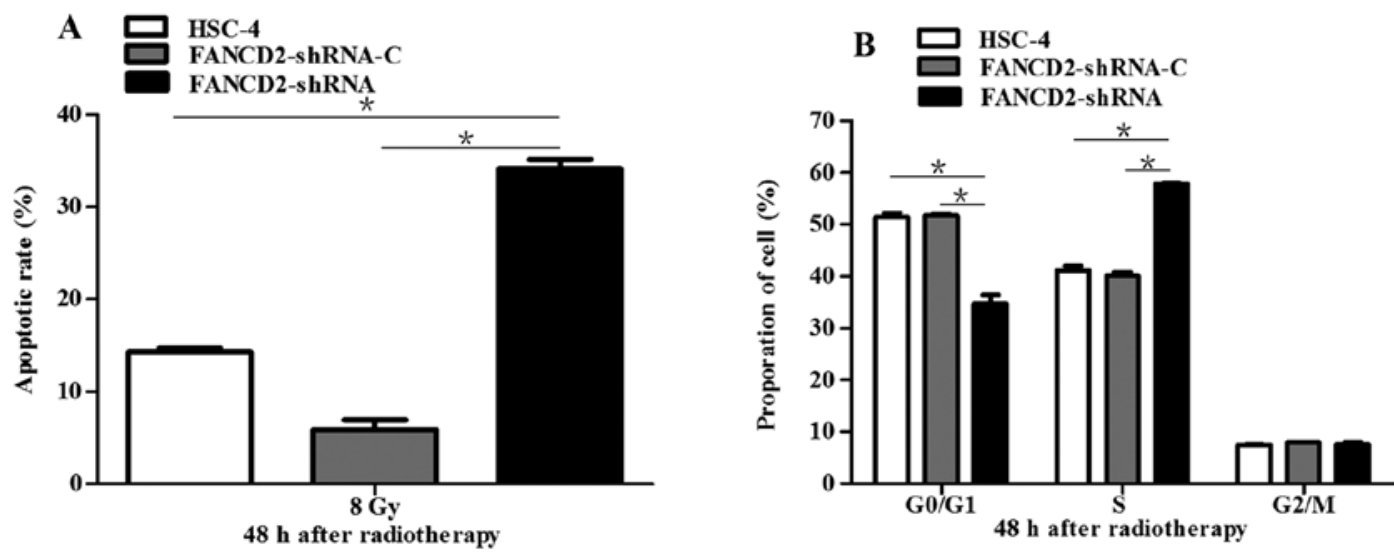

Figure 4. Silencing of FANCD2 enhanced the radiotherapy-induced apoptosis of HSC-4 cells and induced S phase arrest. (A) Apoptosis was evaluated using flow cytometry after the three groups of HSC-4 cells received 8 Gy of radiotherapy. (B) The cell cycle distribution was analyzed by flow cytometry after the three groups of HSC- 4 cells received 8 Gy of radiotherapy. ${ }^{*} \mathrm{P}<0.05$.
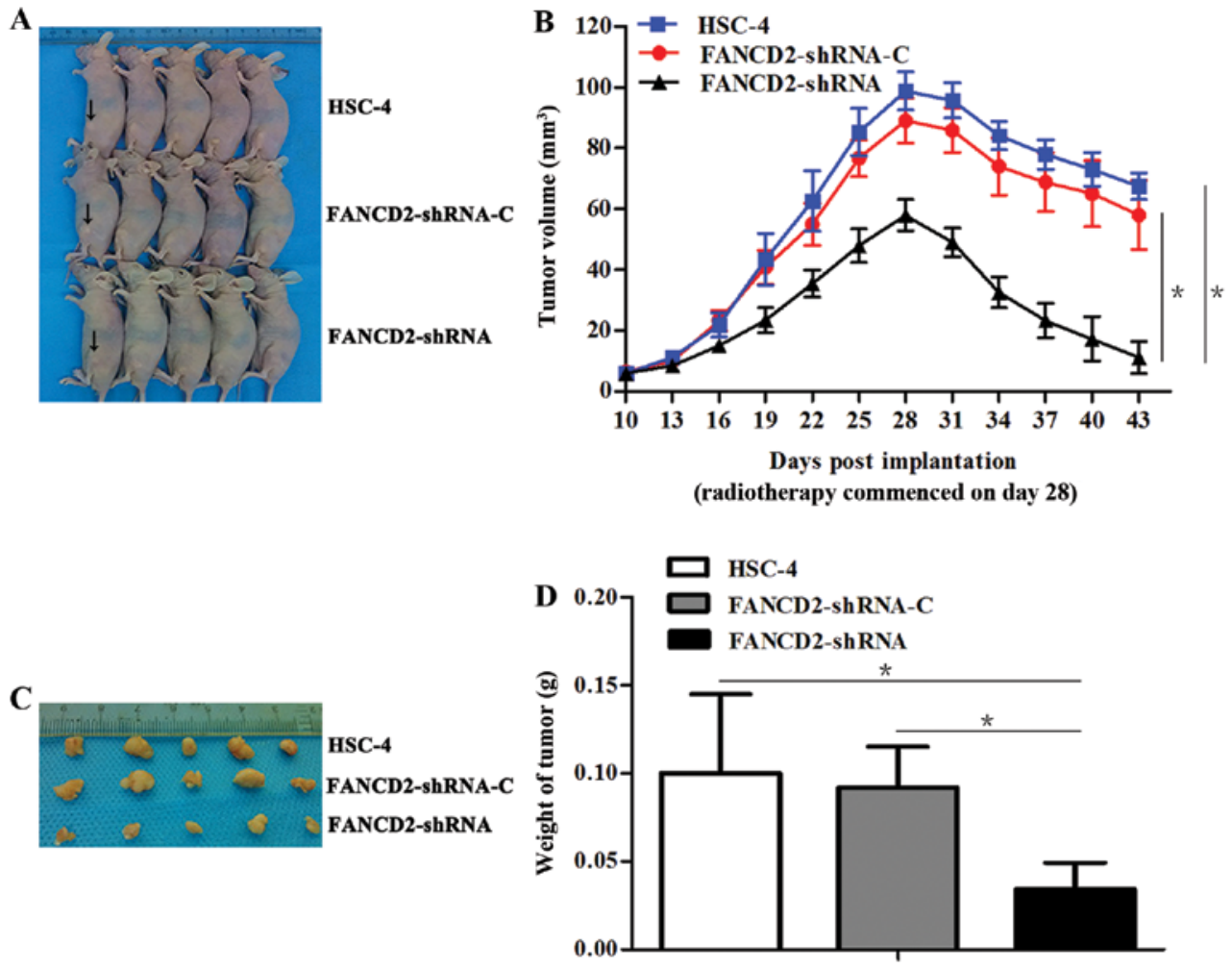

Figure 5. Silencing of FANCD2 by shRNA interference inhibits the growth of tumors derived from transplanted HSC-4 cells. (A) Images of tumor-bearing nude mice 43 days after HSC- 4 cells were transplanted into the three groups of mice. The black arrows indicate the position of the tumors. (B) Changes in the volume of the tumors derived from transplanted HSC-4 cells in the three groups of mice before and after radiotherapy. Before radiotherapy, the growth of the tumors in the FANCD2-shRNA group was significantly slower. Radiotherapy commenced 28 days after the inoculation of the cells, and after radiotherapy, the extent of the decrease in the tumor volume in the FANCD2-shRNA group was significantly greater than that of the negative control group and the blank control group. (C) Images of dissected tumors 43 days after the HSC-4 cells were transplanted into the three groups of mice. (D) A comparison of the tumor weight after HSC-4 cells were transplanted in the mice revealed that the tumor weight in the FANCD2-shRNA group was significantly lower than those in the FANCD2-shRNA-C group and the HSC-4 group. ${ }^{*} \mathrm{P}<0.05$.

Silencing of FANCD2 enhances the radiotherapy-induced apoptosis of HSC-4 cells and induced S phase arrest. A dose of $8 \mathrm{~Gy}$ of radiation was administered to the three groups of cells, and $48 \mathrm{~h}$ later, apoptosis was evaluated using flow cytometry. The result showed that the apoptotic rate of cells in the experimental group was significantly higher than that of cells in the negative and blank control groups $(\mathrm{P}<0.05$; Fig. $4 \mathrm{~A})$, which suggests that the silencing of FANCD2 can increase the radiotherapy-induced apoptotic rate of HSC-4 cells and enhance their sensitivity to radiotherapy. The three groups of cells, which had received a dose of $8 \mathrm{~Gy}$, were cultured under standard conditions for $48 \mathrm{~h}$ after which flow cytometry was used to analyze the cell cycle distribution. The result showed that after radiotherapy, the ratio of cells in $\mathrm{G} 2 / \mathrm{M}$ was not significantly different between the experimental group and the control groups $(\mathrm{P}>0.05)$ but that the proportion of cells in $\mathrm{S}$ 

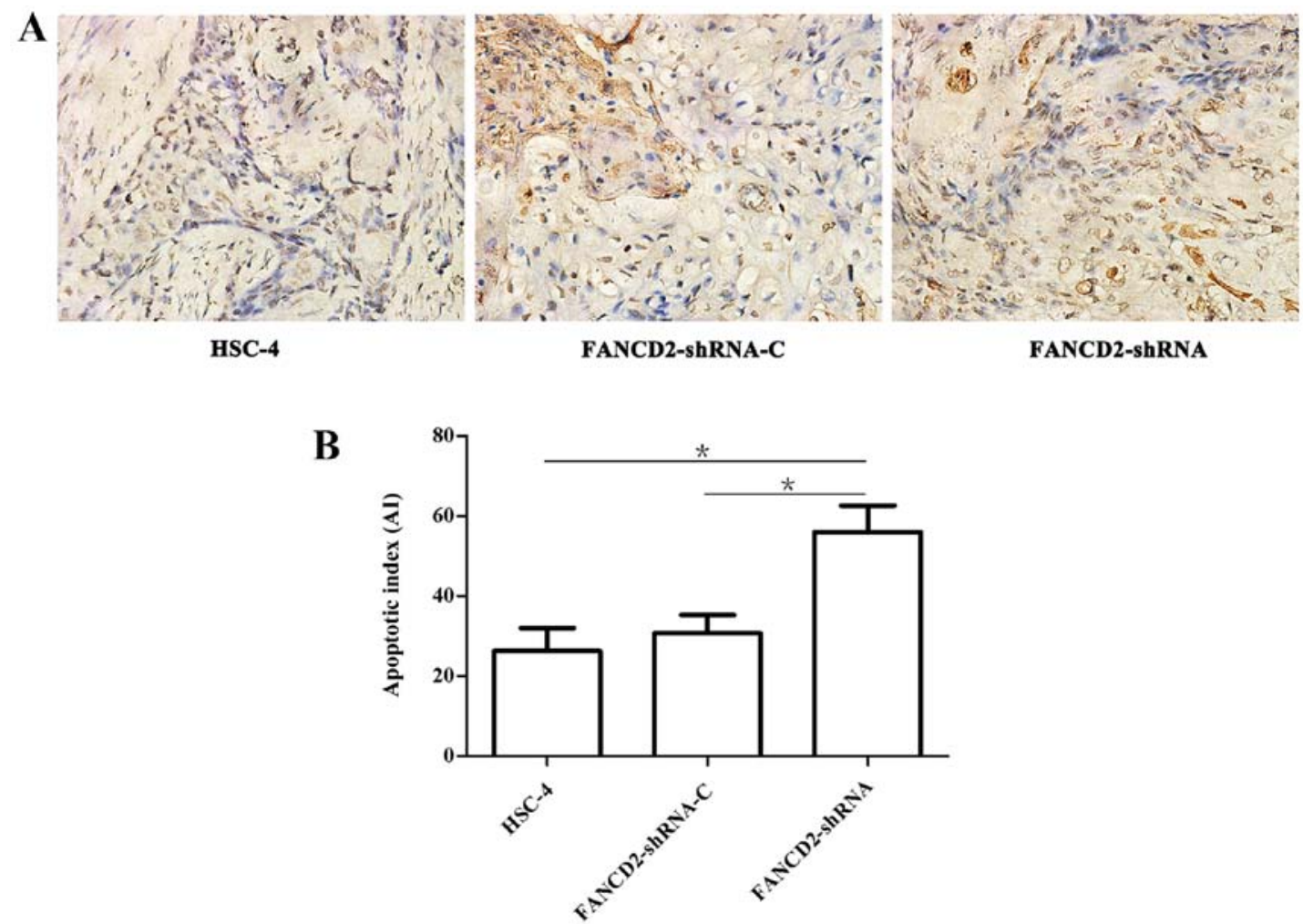

Figure 6. Apoptosis in the tumors derived from HSC-4 cells after radiotherapy. (A) The degree of apoptosis in the three groups of mice with tumors was examined using TUNEL. Light microscopy showed that the nucleus of an apoptotic cell was brown (magnification, $\mathrm{x} 400$ ). (B) The apoptotic rate of the tumors in the FANCD2-shRNA group after radiotherapy was significantly increased compared with the apoptotic rates in the control groups. The AI of the FANCD2shRNA group was also significantly higher than those of the FANCD2-shRNA-C group and the HSC-4 group. "P<0.05.

phase was increased $(\mathrm{P}<0.05)$. In addition, the number of cells entering the G0/G1 phase was decreased ( $\mathrm{P}<0.05$; Fig. 4B), which indicates that the silencing of FANCD2 can induce an $\mathrm{S}$ phase arrest of HSC-4 cells after radiotherapy.

Silencing of FANCD2 inhibits the HSC-4 cell growth in vivo, and increases the sensitivity of tumors to radiotherapy. A tumor mass was visible in all 15 nude mice in the three groups, and the tumor formation rate was $100 \%$ (Fig. 5A). Compared with the control groups, the experimental group, in which tumors were transplanted subcutaneously, demonstrated a prolonged tumor formation time and slowed growth as well as significantly decreased tumor volume and weight. The tumor formation time of HSC-4 cells in the experimental group was 9 to 10 days with a median time of 10 days. The tumor formation time of the negative control group and the blank group was 5 to 7 days with a median time of 6 days. Before radiotherapy, the tumor volume of the experimental group was $57.88 \pm 5.12 \mathrm{~mm}^{3}$, which was significantly lower than that of the negative control group $\left(89.05 \pm 7.44 \mathrm{~mm}^{3}\right)$ and the blank control group $\left(98.83 \pm 6.31 \mathrm{~mm}^{3}\right)(\mathrm{P}<0.05$; Fig. $5 \mathrm{~B})$. After radiotherapy, the tumor volume of the experimental group was $11.11 \pm 5.25 \mathrm{~mm}^{3}$, which was significantly lower than that of the negative control group $\left(57.96 \pm 11.36 \mathrm{~mm}^{3}\right)$ and the blank control group $\left(67.42 \pm 4.40 \mathrm{~mm}^{3}\right)(\mathrm{P}<0.05$; Fig. 5B). Moreover, after radiotherapy, the tumor volume of the experimental group decreased $\left(46.77 \pm 2.76 \mathrm{~mm}^{3}\right)$, which was significantly more than that of the negative control group $\left(31.41 \pm 3.52 \mathrm{~mm}^{3}\right)$ and the blank control group $\left(31.09 \pm 7.69 \mathrm{~mm}^{3}\right)(\mathrm{P}<0.05$; Fig. 5B)
After radiotherapy, the tumor weight of the experimental group was $0.034 \pm 0.015 \mathrm{~g}$, which was significantly lower than that of the negative control group $(0.092 \pm 0.023 \mathrm{~g})$ and the blank control group $(0.100 \pm 0.045 \mathrm{~g})(\mathrm{P}<0.05$; Fig. $5 \mathrm{C}$ and $\mathrm{D})$. Lastly, the tumor weight inhibition rate of the experimental group was $66.00 \pm 15.17 \%$, and the volume inhibition rate was $83.52 \pm 7.78 \%(\mathrm{P}<0.05$; Fig. $5 \mathrm{C}$ and $\mathrm{D})$. These results suggest that the silencing of FANCD2 inhibits the HSC-4 cell growth in vivo and enhances their sensitivity to radiotherapy.

Silencing of FANCD2 increases apoptosis in HSC-4 cell tumors after radiotherapy. A TUNEL assay showed that the three groups of transplanted cells demonstrated different degrees of apoptosis after radiotherapy, but the number of apoptotic cells in the experimental group was significantly higher than that in the negative and the blank control groups (Fig. 6A). The apoptotic index (AI) of the experimental group was $56.02 \pm 6.51 \%$, which was significantly higher than that of the negative control group $(30.80 \pm 4.47 \%)$ and the blank control group $(26.22 \pm 5.82 \%)(\mathrm{P}<0.05$; Fig. $6 \mathrm{~B})$.

Silencing of FANCD2 increased the Bax/Bcl2 ratio and activated the p38 MAPK signaling pathway in HSC-4 cells after radiotherapy. Western blot analysis revealed that after the FANCD2 gene expression in HSC-4 cells was silenced by shRNA interference, the expression of the Bax and p-p38 proteins was significantly higher in the cells of the experimental group than in the control groups $(\mathrm{P}<0.05)$, whereas the expression of the $\mathrm{p} 38$ and $\mathrm{Bcl} 2$ proteins was significantly 
A

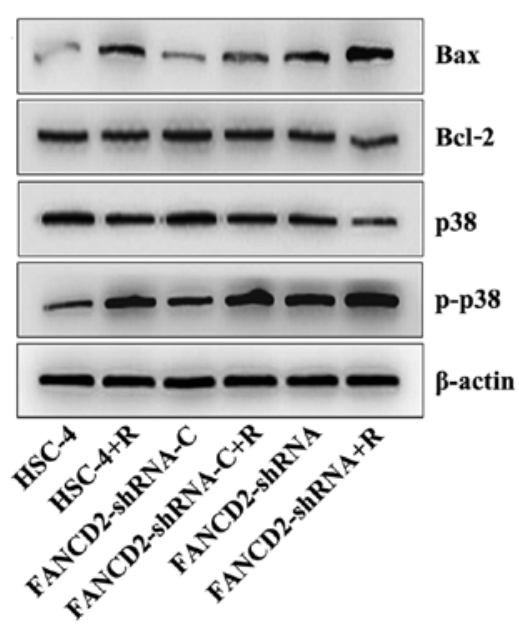

C

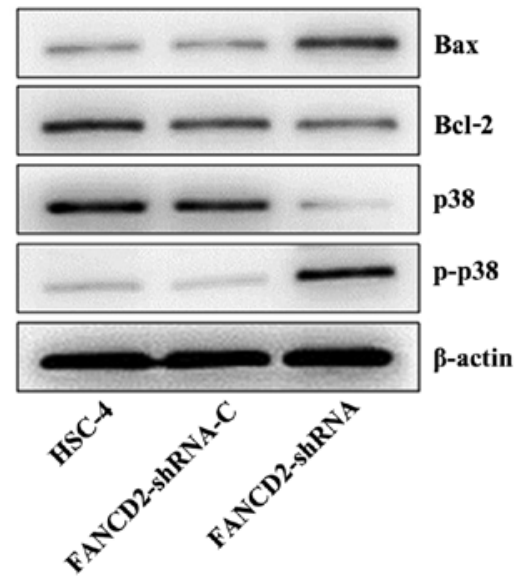

B
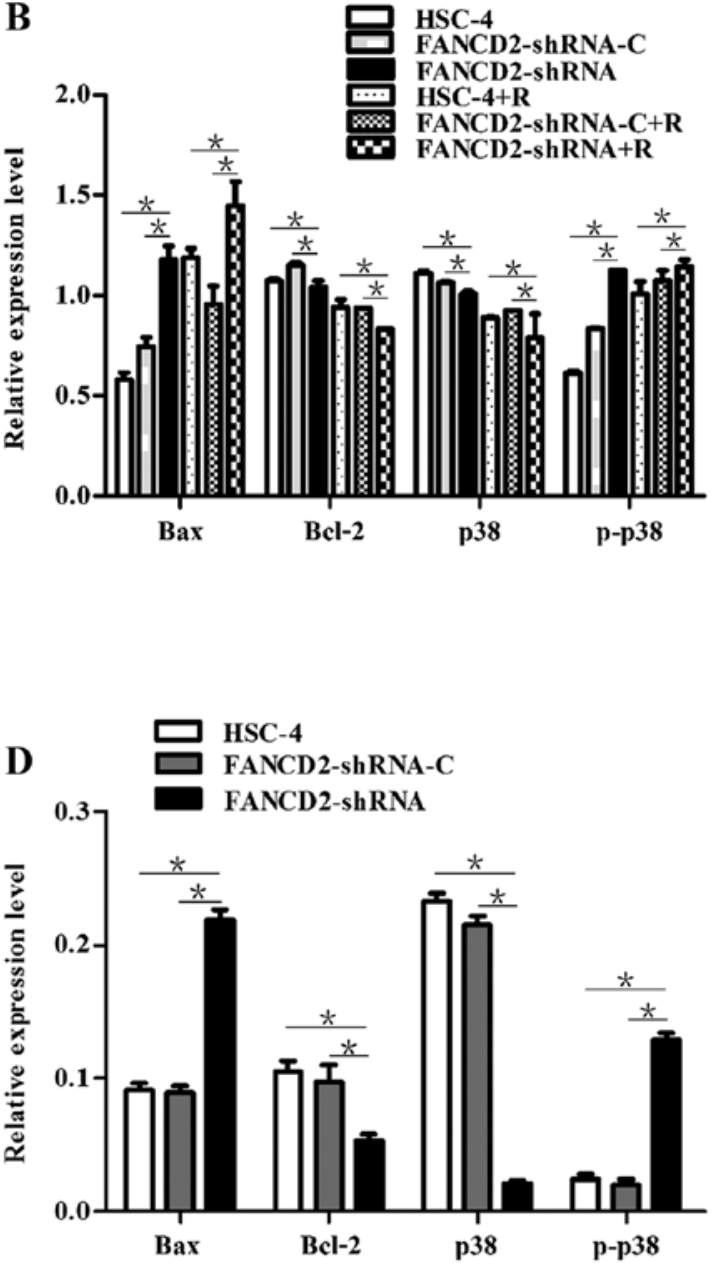

Figure 7. Silencing of FANCD2 increases the Bax/Bcl2 ratio and activates the p38 MAPK signaling pathway in HSC-4 cells after radiotherapy in vitro and in vivo. (A and B) Western blot analysis was used to determine the expression of the Bax, Bcl2, p38 and p-p38 proteins in the three groups of HSC-4 cells before and after radiotherapy. (C and D) Western blot analysis was used to examine the expression of the Bax, Bcl2, p38 and p-p38 proteins in the tumors derived from transplanted HSC-4 cells after radiotherapy. ${ }^{*} \mathrm{P}<0.05$.

decreased $(\mathrm{P}<0.05$; Fig. 7A and B). After radiotherapy, the same results were obtained; additionally, a significant difference was observed in the expression of these proteins before and after radiotherapy $(\mathrm{P}<0.05 ;$ Fig. $7 \mathrm{~A}$ and $\mathrm{B})$. After the nude mice with transplanted HSC-4 cells received radiotherapy, western blotting and immunohistochemistry were performed, which showed an increase in the expression of Bax and p-p38 proteins in the tumor tissues of mice in the experimental group compared with the control group $(\mathrm{P}<0.05)$; in contrast, the expression of $\mathrm{p} 38$ and $\mathrm{Bcl} 2$ proteins was decreased $(\mathrm{P}<0.05$; Figs. 7C and D, 8 and 9). This finding is consistent with the results of the in vitro cell culture experiments. These results suggest that the silencing of FANCD2 increases the Bax/Bcl2 ratio and activates the p38 MAPK signaling pathway in HSC-4 cells after radiotherapy.

\section{Discussion}

A study by Lyakhovich et al (16) found that the transient silencing of FANCD2 expression using siRNA interference could increase the sensitivity of breast cancer cells, liver cancer cells and bladder cancer cells to $\gamma$-rays and DNA damaging agents, and such an enhanced sensitivity effect is closely associated with the decrease in the proliferation ability of cancer cells. The present study found that the three groups of cells that received radiotherapy had a lower proliferation rate compared with the group that did not receive radiotherapy. The proliferation inhibition effect was enhanced as the radiation dose increased, and the cell proliferation rate was decreased as the radiotherapy duration increased; these effects were most significant in the FANCD2 knockdown group. A colony formation assay also confirmed that the cell survival rate of the experimental group after radiotherapy was significantly lower than that of the negative and the blank control groups. This finding suggests that the enhancement in the sensitivity of HSC-4 cells to radiotherapy by FANCD2 silencing is associated with a decrease in cell proliferation ability.

A large number of studies have found that cell cycle regulation has a significant impact on the survival of tumor cells and their sensitivity to radiotherapy, where G2/M phase arrest can 

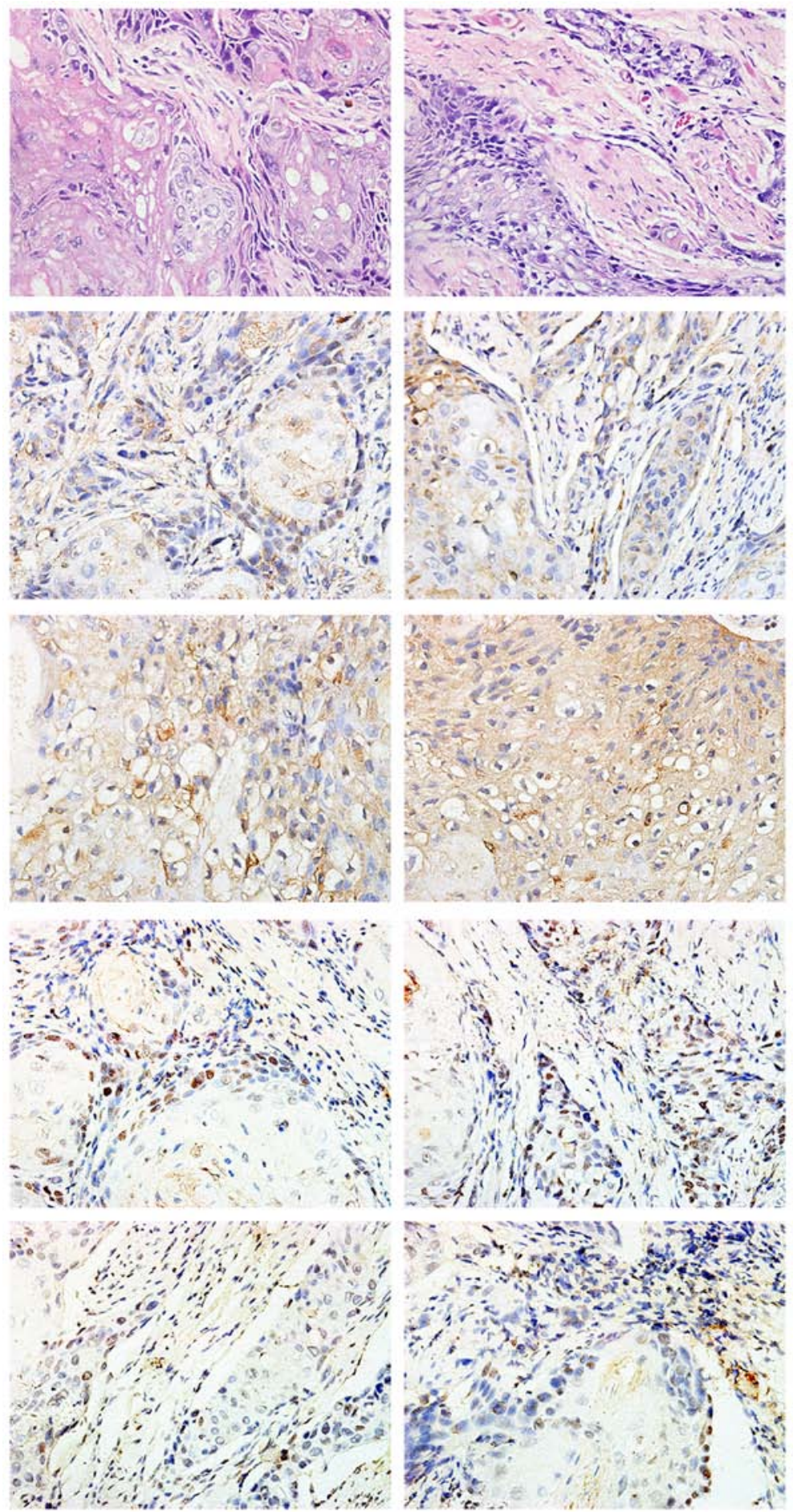

HSC-4
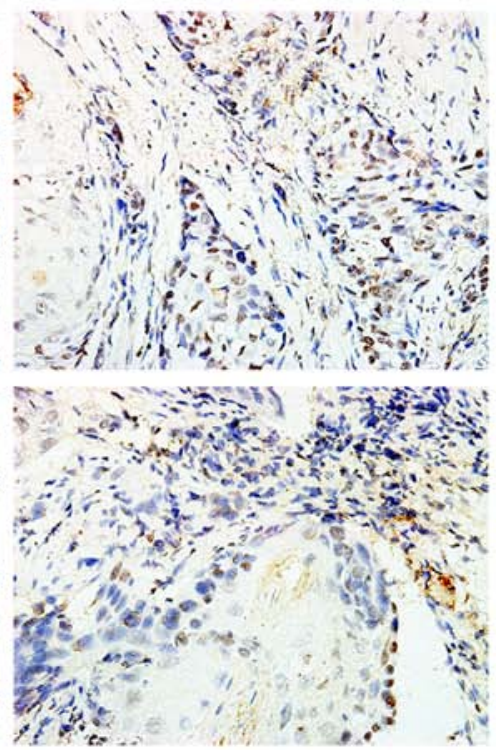

FANCD2-shRNA-C

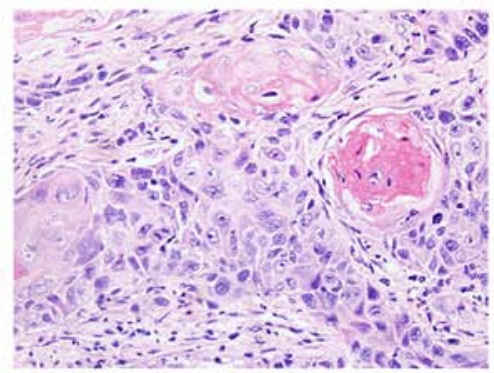

H\&E

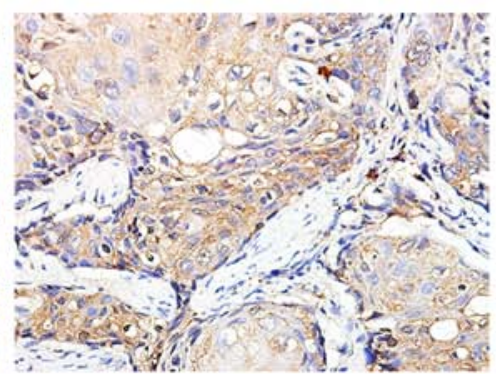

Bax

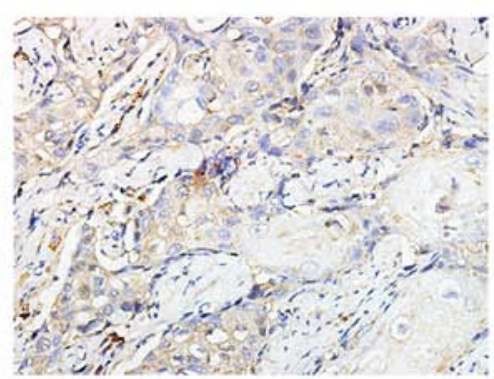

Bcl-2
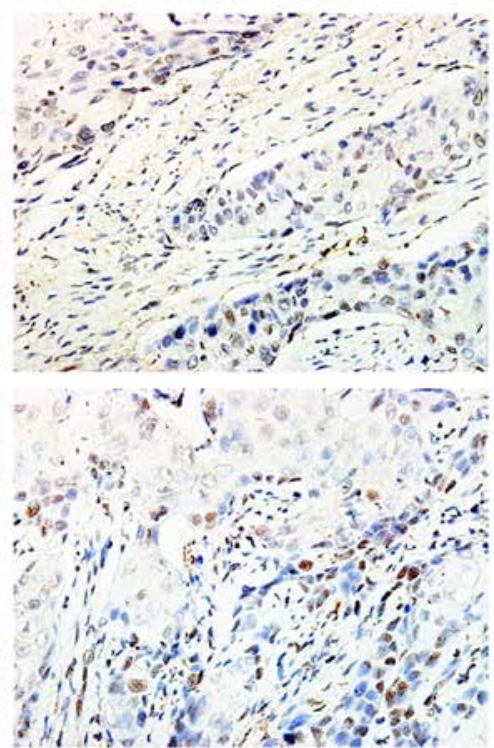

FANCD2-shRNA

Figure 8. H\&E staining of the tumors derived from HSC-4 cells and immunohistochemical analysis of the Bax, Bcl2, p38 and p-p38 protein expression after radiotherapy. Conventional histopathological H\&E staining showed that the three groups of tumors demonstrated the characteristics of squamous cell carcinoma (magnification, $\mathrm{x} 400$ ). Positive expression of Bax and Bcl2 was found in the cytoplasm, and positive expression of p38 was primarily found in the cytoplasm but also partly in the nucleus. Positive expression of p-p38 was observed only in the nucleus (magnification, $\mathrm{x} 400$ EnVision method).

enhance the sensitivity of tumor cells to radiotherapy (17-19). The present study found that after radiotherapy, the $\mathrm{G} 2 / \mathrm{M}$ phase ratio of HSC-4 cells in the experimental group was not significantly different compared with that in the control groups. In addition, the proportion of cells in $\mathrm{S}$ phase increased significantly, and the number of cells entering the G0/G1 phase decreased, which does not seem to correspond to an increased proportion of cells in the G2/M phase. However, studies by other scholars found that the inhibition of Bcl2 function could promote the apoptosis of HNSCC cells, which results in S phase arrest (20). Therefore, the increase in the proportion of cells in $S$ phase after radiotherapy in the experimental group, which was found in the present study, may be related to the downregulation of $\mathrm{Bcl} 2$ expression. Furthermore, ubiquitination, which is 


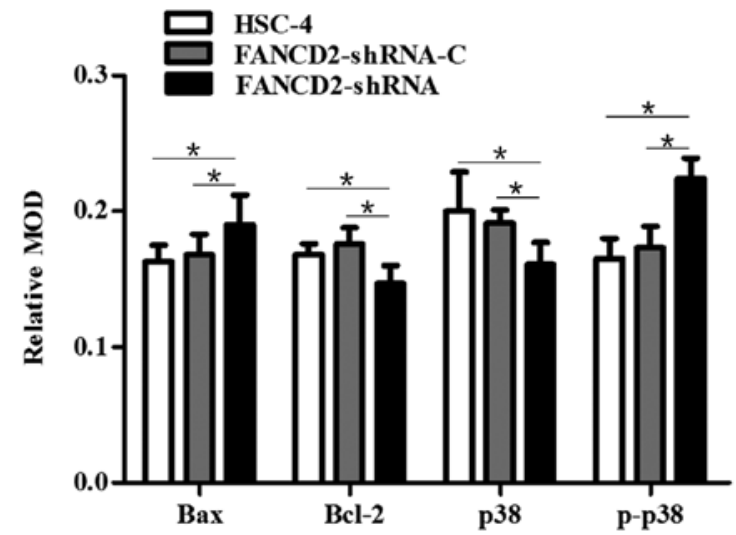

Figure 9. Image-Pro Plus 6 software was used to quantitatively analyze the expression of the $\mathrm{Bax}, \mathrm{Bcl} 2, \mathrm{p} 38$ and $\mathrm{p}-\mathrm{p} 38$ proteins using immunohistochemistry (IHC) in the tumor sections from the three groups of mice after radiotherapy. After radiotherapy, the mean optical density (MOD) values for the expression of the Bax and p-p38 proteins in the FANCD2-shRNA group were significantly higher than those in the FANCD2-shRNA-C and HSC-4 groups. In contrast, the MOD values for the expression of the $\mathrm{Bcl} 2$ and p38 proteins were significantly lower than those in the FANCD2-shRNA-C group and the HSC-4 group. ${ }^{*} \mathrm{P}<0.05$

important for the repair of DNA damage caused by radiotherapy, occurs in the $\mathrm{S}$ phase of the cell cycle. The downregulation of FANCD2 reduced the ubiquitination level of FANCD2, which affects the DNA damage repair process and causes cells to arrest in $S$ phase (21).

Apoptosis is one of the important factors in the radiosensitivity of tumor cells; and it is regulated by a multiple-gene network, of which the $\mathrm{Bax}$ and $\mathrm{Bcl} 2$ gene families have great importance in the molecular regulation of apoptosis. Farnebo et al (22) found that Bax, Bcl2, Bcl-X (L), COX-2 and p53 were closely related to the predicted radiosensitivity of HNSCC cell lines. Some scholars reported that the radiationinduced reduction in $\mathrm{Bcl} 2$ and elevation in Bax were associated with an increase in the radiosensitivity of tumor cells (23). Studies by Oltvai et al (24) demonstrated that $\mathrm{Bax}$ and $\mathrm{Bcl} 2$ existed in the form of a dimer in cells. When the $\mathrm{Bcl} 2$ protein was expressed in excess, $\mathrm{Bcl} 2 / \mathrm{Bcl} 2$ homodimers were formed, inhibiting apoptosis; however, when the expression of the Bax protein was excessive, Bax/Bax homodimers were formed, which also promoted apoptosis. Studies indicated that an increase in Bax protein expression could induce an increase in apoptosis via the activation of the caspase signal transduction pathway (25). In the present study, both in vivo and in vitro experiments showed that apoptosis induced by radiotherapy in the experimental group was significantly increased compared with that of the negative and the blank control groups. Moreover, a western blot analysis found that after FANCD2 expression was silenced by shRNA interference, Bax expression was increased in the experimental group compared with the control groups. In contrast, $\mathrm{Bcl} 2$ expression was decreased in the experimental group compared with the control groups. After the three groups of cells received radiotherapy, Bax expression was increased in the shRNA interference group, while $\mathrm{Bcl} 2$ expression was reduced; this difference in protein expression was significant before and after treatment with radiotherapy. Thus, we speculate that the silencing of FANCD2 can enhance radiosensitivity through the promotion of radiotherapy-induced apoptosis in HSC-4 cells, which is consistent with the experimental results of Van Der Heijden et al (26). We also speculate that this mechanism may be related to the expression of $\mathrm{Bax}$ and $\mathrm{Bcl} 2$, which regulate the apoptosis pathway in mitochondria.

p38 mitogen-activated protein kinase (MAPK) is one of the members of the highly conserved threonine/serine mitogenactivated protein kinase family, and studies have confirmed that p38 MAPK is involved in apoptosis, cell differentiation, the cell cycle and cell senescence (27). The inhibition of the activation of the $\mathrm{p} 38$ signal transduction pathway can induce apoptosis (28). p38 MAPK could promote apoptosis of lung adenocarcinoma cells via the regulation of the expression of the apoptosis-related proteins Bax and Bcl2 (29). The downregulation of p38 MAPK could reverse the radiotherapy resistance caused by the overexpression of miRNA (30). The present study found that after FANCD2 was silenced by shRNA interference, the expression of the p38 protein in the cells of the experimental group was decreased, while the expression of the p-p38 protein was increased compared with the expression in the control groups. After radiotherapy, the expression of the p38 and p-p38 proteins in the HSC-4 cells in the experimental group showed a consistent trend similar to that observed before radiotherapy. This difference in protein expression before and after radiotherapy was significant, which was consistent with the results of animal experiments. Therefore, the silencing of FANCD2 can enhance the radiotherapy-induced apoptosis of HSC- 4 cells via the regulation of the expression of p38 MAPK pathway-related proteins, leading to increased radiosensitivity.

In summary, the present study confirms that shRNA can stably knock down FANCD2 expression in HSC-4 cells. In both in vivo and in vitro experiments, we found that the silencing of FANCD2 by shRNA interference enhances the sensitivity of metastatic lymph node-derived HSC-4 HNSCC cells to radiotherapy. We demonstrate that the mechanism of increased radiosensitivity may be related to the activation of the p38 MAPK signaling pathway and the regulation of the expression of the apoptosis-related proteins $\mathrm{Bax}$ and $\mathrm{Bcl} 2$, which has provided potential new molecular targets for the treatment of HNSCC. Finally, this study provided a theoretical basis for further clinical studies.

\section{Acknowledgements}

The present study was supported by grants from the Major Project of Education Department of Sichuan Province (12ZA076), the Health and Family Planning Commission of Sichuan Province (120352), the Scientific Research Project of the Affiliated Hospital of Luzhou Medical College for Returned Chinese Scholars (2013-60-2), the Scientific Research Project of the Southwest Medical University, and the Joint Research Project of Science Technology Department of Sichuan Province - Office of Science Technology of Luzhou - Luzhou Medical College (14JC0182), China.

\section{References}

1. Wise-Draper TM, Draper DJ, Gutkind JS, Molinolo AA, Wikenheiser-Brokamp KA and Wells SI: Future directions and treatment strategies for head and neck squamous cell carcinomas. Transl Res 160: 167-177, 2012. 
2. Lee JH, Song JH, Lee SN, Kang JH, Kim MS, Sun DI and Kim YS: Adjuvant postoperative radiotherapy with or without chemotherapy for locally advanced squamous cell carcinoma of the head and neck: The importance of patient selection for the postoperative chemoradiotherapy. Cancer Res Treat 45: 31-39, 2013.

3. Argiris A, Karamouzis MV, Raben D and Ferris RL: Head and neck cancer. Lancet 371: 1695-1709, 2008.

4. Thariat J, Milas L and Ang KK: Integrating radiotherapy with epidermal growth factor receptor antagonists and other molecular therapeutics for the treatment of head and neck cancer. Int J Radiat Oncol Biol Phys 69: 974-984, 2007.

5. Kupfer GM: Fanconi anemia: A signal transduction and DNA repair pathway. Yale J Biol Med 86: 491-497, 2013.

6. Kutler DI, Auerbach AD, Satagopan J, Giampietro PF, Batish SD, Huvos AG, Goberdhan A, Shah JP and Singh B: High incidence of head and neck squamous cell carcinoma in patients with Fanconi anemia. Arch Otolaryngol Head Neck Surg 129: 106-112, 2003.

7. Virts EL, Jankowska A, Mackay C, Glaas MF, Wiek C, Kelich SL, Lottmann N, Kennedy FM, Marchal C, Lehnert E, et al: AluYmediated germline deletion, duplication and somatic stem cell reversion in UBE2T defines a new subtype of Fanconi anemia. Hum Mol Genet 24: 5093-5108, 2015.

8. Rickman KA, Lach FP, Abhyankar A, Donovan FX, Sanborn EM, Kennedy JA, Sougnez C, Gabriel SB, Elemento O, Chandrasekharappa SC, et al: Deficiency of UBE2T, the E2 ubiquitin ligase necessary for FANCD2 and FANCI ubiquitination, causes FA-T subtype of Fanconi anemia. Cell Rep 12: 35-41, 2015.

9. Kee $\mathrm{Y}$ and D'Andrea AD: Molecular pathogenesis and clinical management of Fanconi anemia. J Clin Invest 122: 3799-3806, 2012.

10. Kim H and D'Andrea AD: Regulation of DNA cross-link repair by the Fanconi anemia/BRCA pathway. Genes Dev 26 : 1393-1408, 2012.

11. Jo U and Kim H: Exploiting the Fanconi anemia pathway for targeted anti-cancer therapy. Mol Cells 38: 669-676, 2015.

12. Wang LC and Gautier J: The Fanconi anemia pathway and ICL repair: Implications for cancer therapy. Crit Rev Biochem Mol Biol 45: 424-439, 2010.

13. Burkitt $\mathrm{K}$ and Ljungman M: Phenylbutyrate interferes with the Fanconi anemia and BRCA pathway and sensitizes head and neck cancer cells to cisplatin. Mol Cancer 7: 24, 2008.

14. Zhu J, Su F, Mukherjee S, Mori E, Hu B and Asaithamby A: FANCD2 influences replication fork processes and genome stability in response to clustered DSBs. Cell Cycle 14: 1809-1822, 2015.

15. Burdak-Rothkamm S, Rothkamm K, McClelland K, Al Rashid ST and Prise KM: BRCA1, FANCD2 and Chk1 are potential molecular targets for the modulation of a radiationinduced DNA damage response in bystander cells. Cancer Lett 356 (2 Pt B): 454-461, 2015.

16. Lyakhovich A and Surralles J: FANCD2 depletion sensitizes cancer cells repopulation ability in vitro. Cancer Lett 256 : 186-195, 2007.
17. Jeong YK, Kim MS, Lee JY, Kim EH and Ha H: Metformin radiosensitizes p53-deficient colorectal cancer cells through induction of $\mathrm{G} 2 / \mathrm{M}$ arrest and inhibition of DNA repair proteins. PLoS One 10: e0143596, 2015.

18. Liu J, Ge YY, Zhu HC, Yang X, Cai J, Zhang C, Lu J, Zhan LL, Qin Q, Yang Y, et al: Fenofibrate increases radiosensitivity in head and neck squamous cell carcinoma via inducing G2/M arrest and apoptosis. Asian Pac J Cancer Prev 15: 6649-6655, 2014.

19. Duangmano S, Sae-Lim P, Suksamrarn A, Patmasiriwat P and Domann FE: Cucurbitacin B causes increased radiation sensitivity of human breast cancer cells via G2/M cell cycle arrest. J Oncol 2012: 601682, 2012.

20. Ashimori N, Zeitlin BD, Zhang Z, Warner K, Turkienicz IM, Spalding AC, Teknos TN, Wang S and Nör JE: TW-37, a smallmolecule inhibitor of Bcl-2, mediates S-phase cell cycle arrest and suppresses head and neck tumor angiogenesis. Mol Cancer Ther 8: 893-903, 2009.

21. Taniguchi T, Garcia-Higuera I, Andreassen PR, Gregory RC, Grompe $M$ and D'Andrea AD: S-phase-specific interaction of the Fanconi anemia protein, FANCD2, with BRCA1 and RAD51. Blood 100: 2414-2420, 2002.

22. Farnebo L, Jerhammar F, Ceder R, Grafström RC, Vainikka L, Thunell L, Grénman R, Johansson AC and Roberg K: Combining factors on protein and gene level to predict radioresponse in head and neck cancer cell lines. J Oral Pathol Med 40: 739-746, 2011.

23. Findley HW, Gu L, Yeager AM and Zhou M: Expression and regulation of $\mathrm{Bcl}-2, \mathrm{Bcl}-\mathrm{xl}$, and $\mathrm{Bax}$ correlate with p53 status and sensitivity to apoptosis in childhood acute lymphoblastic leukemia. Blood 89: 2986-2993, 1997.

24. Oltvai ZN, Milliman CL and Korsmeyer SJ: Bcl-2 heterodimerizes in vivo with a conserved homolog, Bax, that accelerates programmed cell death. Cell 74: 609-619, 1993.

25. Schon EA and Manfredi G: Neuronal degeneration and mitochondrial dysfunction. J Clin Invest 111: 303-312, 2003.

26. Van Der Heijden MS, Brody JR and Kern SE: Functional screen of the fanconi anemia pathway in cancer cells by Fancd 2 immunoblot. Cancer Biol Ther 3: 534-537, 2004.

27. Raman M, Chen $\mathrm{W}$ and Cobb MH: Differential regulation and properties of MAPKs. Oncogene 26: 3100-3112, 2007.

28. Chun J, Joo EJ, Kang M and Kim YS: Platycodin D induces anoikis and caspase-mediated apoptosis via p38 MAPK in AGS human gastric cancer cells. J Cell Biochem 114: 456-470, 2013.

29. Su JC, Lin KL, Chien CM, Lu CM, Chen YL, Chang LS and Lin SR: Novel indoloquinoline derivative, IQDMA, induces $\mathrm{G}(2) / \mathrm{M}$ phase arrest and apoptosis in A549 cells through JNK/ p38 MAPK signaling activation. Life Sci 85: 505-516, 2009.

30. Salim H, Akbar NS, Zong D, Vaculova AH, Lewensohn R, Moshfegh A, Viktorsson K and Zhivotovsky B: miRNA-214 modulates radiotherapy response of non-small cell lung cancer cells through regulation of p38MAPK, apoptosis and senescence. Br J Cancer 107: 1361-1373, 2012. 